Im Journal Club referieren und kommentieren diese Experten für Sie die wichtigsten Arbeiten aus der internationalen Fachliteratur.

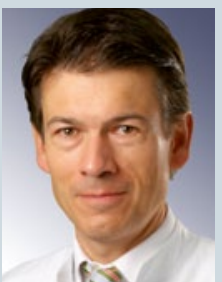

Prof. Dr. med. Peter Mallmann, Köln

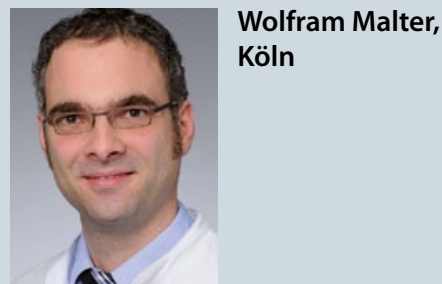

\title{
Keine Assoziation von Blutgruppe und Mammakarzinom
}

\section{Studien haben einen Zusammenhang zwischen der ABO-Blutgruppe und verschiedenen Tumoren gezeigt. Beim Mammakarzinom waren die bisherigen Ergebnisse inkonsistent. Jetzt wurde in einer sehr umfangreichen Studie belegt, dass zwischen der serologischen wie auch phänotypischen ABO-Blutgruppe und den verschiedenen Formen des Mammakarzinoms keine Verbindung besteht.}

E pidemiologische Studien zeigten, dass _die ABO-Blutgruppe mit dem Risiko und der Überlebensrate bei verschiedenen Tumoren assoziiert ist. Beim Mammakarzinom dagegen ist die Datenlage hinsichtlich eines Zusammenhangs bisher nicht eindeutig geklärt. Ältere Studien fanden keinen Zusammenhang, eine neue Untersuchung wies auf ein Risiko für ein duktales Mammakarzinom bei der Blutgruppe A hin. Zwei weitere Studien belegten eine Assoziation zwischen der Blutgruppe A oder B und einer erhöhten Rate an familiären Brustkrebs. Keine eindeutigen Aussagen ließen sich für den Rhesus-Faktor und die Überlebensrate bezogen auf die ABO-Blutgruppe finden. Das auf dem Chromosom 9q34 lokali-

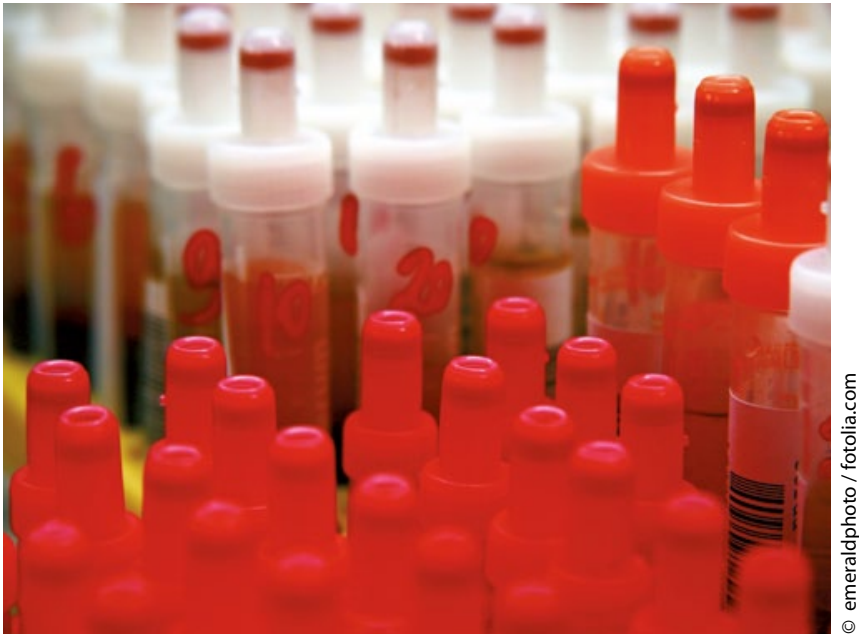

sierte ABO-Gen kodiert für die Glykosyltransferasen. Die Blutgruppe A- und B-Isoantigene lassen sich auf der Oberfläche normaler duktaler Mamma-Zellen und auch auf normalen lobulären Zellen des Brustgewebes nachweisen. Es wurde gezeigt, dass es bei manchen Mammakarzinomen zum Verlust der ABO-AntigenExpression kommt, was auf eine mögliche Rolle in der Karzinogenese hinweisen könnte.

In einer großen, prospektiven Studie aus den USA wurde die Blutgruppe und die Inzidenz sowie die Überlebensrate beim Mammakarzinom im Rahmen der Nurses ' Health Study untersucht. Das untersuchte Kollektiv bestand aus 67.697 Frauen mit 3.107 Fällen eines inziden-

Eine groß angelegte Studie klärte es nun: Es besteht kein Zusammenhang zwischen Blutgruppe und Brustkrebsrisiko. ziellen Mammakarzinoms. Die serologische und/oder genotypische Blutgruppe war den teilnehmenden Frauen bekannt. Mittels proportionaler Cox-Regressionsanalyse wurde der Zusammenhang mit der serologischen Blutgruppe untersucht. Zusätzlich erfolgte eine geschachtelte FallKontroll-Analyse zwischen der phänotypischen Blutgruppe und dem invasiven Mammakarzinom bei 1.138 erkrankten Frauen und 1.090 Kontrollen. Ferner wurde geprüft, ob ein Zusammenhang zwischen der serologischen Blutgruppe und dem Überleben bei 2.036 Frauen mit Mammakarzinom besteht.

Nach Auswertung der Ergebnisse ließ sich kein statistischer Zusammenhang zwischen der serologischen und phänotypischen $\mathrm{ABO}$-Blutgruppe und dem Risiko der verschiedenen Formen des Mammakarzinoms erkennen.

Fazit: Durch diese umfangreiche prospektive Studie, die zugleich die erste zu diesem Thema war, konnte jetzt endgültig die Frage beantwortet werden, ob zwischen dem Mammakarzinom und der Blutgruppe ein Zusammenhang besteht. Die Antwort lautet: "Nein". Doch auch negative Ergebnisse wie in dieser Studie sind hinsichtlich der grundsätzlichen Erkenntnis von Bedeutung.

Prof. Dr. Tino F. Schwarz

Gates MA et al.: ABO blood group and breast cancer incidence and survival. Int. J. Cancer 2012; 130: 2129-37 